\section{Les aérosols-doseurs contenant des CFC seront bientôt interdits}

C. $A b t$
La densité de la couche d'ozone a diminué d'environ $10 \%$ depuis le début des années 70 , entraînant une augmentation du rayonnement UV d'environ $15 \%$. Le trou dans la couche d'ozone constitue la manifestation la plus spectaculaire de cette évolution: en automne 2000, il atteignait la taille record de 29,3 millions de kilomètres carrés, soit plus de trois fois la surface de l'Europe.

En 1987, 25 états, dont la Suisse, ont signé le "Protocole de Montréal» relatif aux substances qui appauvrissent la couche d'ozone. Cet accord, que 150 états ont maintenant signé, interdit la production et l'utilisation des CFC. Leur production n'est plus autorisée aujourd'hui que pour la fabrication des aérosols-doseurs utilisés en médecine. Les CFC ne devront plus être produits - et ne seront donc plus disponibles - à partir de 2005.

Toutes les émissions de CFC sont dangereuses pour la couche d'ozone. Leur utilisation à des fins médicales représente quelque 10000 tonnes par an dans le monde, soit $0,5 \%$ des quantités de CFC produites à l'origine. Les aérosols-doseurs devront utiliser des gaz propulseurs autres que les CFC ou être remplacés par d'autres formes de thérapies.

Aujourd'hui, en Suisse, plusieurs fabricants d'aérosols-doseurs ont déjà abandonné les CFC pour un autre gaz propulseur. D'autres s'apprêtent à le faire.

1930 dans les appareils de réfrigération et de climatisation ainsi que dans les aérosols les plus divers, dans le monde entier. En raison de leurs propriétés, ils sont également employés comme gaz propulseur dans les aérosols médicamenteux. Depuis 1956, un grand nombre de principes actifs servant à traiter les affections des voies respiratoires sont appliqués au moyen d'aérosols-doseurs contenant des CFC.

Les aérosols-doseurs constituent le système le plus utilisé à l'échelle du globe pour administrer les principes actifs nécessaires au traitement des maladies des voies respiratoires. Environ 70 millions de patients d'une centaine de pays s'en servent, ce qui représente quelque 340 millions d'aérosols-doseurs chaque année.

Depuis bien longtemps, les scientifiques ont constaté que la couche d'ozone souffrait de l'utilisation des CFC. Cette couche se trouve à une hauteur de 15 à $30 \mathrm{~km}$ au-dessus de la surface de la terre, à l'intérieur de ce qu'on appelle la stratosphère. Elle nous protège du rayonnement direct des ultraviolets et absorbe notamment les ultraviolets B et C, extrêmement dangereux. Sans la couche d'ozone, il n'y aurait pas de vie sur terre, comme c'était le cas il y a des millions d'année. Un rayonnement UV croissant favorise les cancers de la peau, affaiblit le système immunitaire et provoque diverses maladies des yeux. Il empêche également la croissance des plantes et des petits organismes aquatiques.

Correspondance:

Dr Carine Abt

Im Neusatz 2

CH-4144 Arlesheim

\section{Gaz propulseurs de substitution}

Le gaz propulseur de substitution ne doit pas seulement avoir les mêmes propriétés que les CFC (non toxique, non réactif, non inflammable, inerte, sans goût, etc.), mais aussi présenter les mêmes caractéristiques physiques et chimiques. Les sociétés pharmaceutiques concernées se sont réunies sous la forme d'un consortium pour leurs travaux de recherche et de développement d'un tel gaz propulseur.

Les gaz propulseurs disponibles aujourd'hui en Suisse, ou qui le seront prochainement, sont des alcanes fluorés, HFA-134a ou HFA 227.

Les alcanes fluorés n'ont certes aucun effet sur la couche d'ozone, mais ils subsistent des dizaines d'années dans l'atmosphère et présentent un potentiel de réchauffement considérable (1300 à 3300 fois celui $\mathrm{du} \mathrm{CO}_{2}$ ). En raison de leur potentiel d'effet de serre, les gaz propulseurs fluorés ne constituent donc qu'une solution transitoire.

\section{Possibilités thérapeutiques}

Actuellement, l'inhalation fait partie intégrante du traitement standard des affections aiguës et chroniques des voies respiratoires. Le médicament est inhalé soit sous la forme d'une solution, à l'aide d'un inhalateur, soit au moyen d'aérosols-doseurs ou d'inhalateurs de poudre faciles à utiliser. Il parvient ainsi directement dans les bronches et les poumons. 
Solutions à inhaler

La solution est inhalée à l'aide d'un inhalateur (p. ex. Pari). L'avantage est que cette solution peut être conçue en fonction des besoins individuels du patient, sur ordonnance. Les solutions inhalées se prêtent particulièrement aux nourrissons et aux jeunes enfants ainsi qu'aux personnes âgées.

D'après la Ligue pulmonaire suisse, plus de 28000 inhalateurs sont utilisés dans notre pays.

\section{Aérosols-doseurs}

Dans l'aérosol-doseur, le médicament se présente soit dissous, soit en suspension dans le gaz propulseur liquide. Au moment de la pulvérisation, il est nébulisé en fines gouttelettes ou particules ou réduit en poudre et se présente sous cette forme dans l'aérosol. Une certaine quantité du médicament ou de son principe actif est alors libérée.

Aujourd'hui, les aérosols-doseurs contiennent des gaz propulseurs fluorés en lieu et place des CFC. Le passage des CFC aux alcanes fluorés est un processus compliqué et coûteux. Il faut non seulement trouver la composition idéale des principes actifs et excipients, mais aussi développer de nouveaux mécanismes de spray. Les études cliniques doivent parfois être répétées pour l'enregistrement afin de prouver que le rapport utilité-risque est aussi bon que pour les aérosols contenant des CFC. Mais il n'est pas exclu que le passage à des aérosols sans CFC réclame une adaptation de la dose, en fonction de la préparation et de l'individu. L'objectif est de remplacer tous les aérosols-doseurs contenant des CFC par d'autres aérosols-doseurs au cours des prochaines années.

\section{Inhalateurs de poudre}

L'industrie pharmaceutique a travaillé intensivement, au cours des 15 dernières années, à la recherche de nouvelles substances pour soigner les maladies des voies respiratoires, mais aussi à de nouvelles technologies d'inhalation. La grande innovation, due assurément à la menace de l'interdiction des aérosols propulsés par CFC, a abouti à une multitude de nouveaux inhalateurs de poudre, tels que "Diskhaler», "Turbuhaler», "Diskus» et "Aerolizer».

L'inhalation de poudre a non seulement été développée pour des raisons de protection de l'environnement, mais aussi pour mieux acheminer le principe actif vers les poumons. Etant donné que la poudre doit être aspirée, la coordination problématique que réclame l'aérosol-doseur - pulvériser et inspirer - disparaît.

L'inhalation de poudre s'est surtout imposée dans les pays conscients de l'importance de l'environnement. En Suède, 90\% des thérapies sont appliquées par inhalateurs de poudre. En Suisse, actuellement, le pourcentage est de $40 \%$.

\section{Conséquences et recommandations}

Aucun changement n'intervient pour les patients utilisant déjà des inhalateurs de poudre.
Pour des raisons de protection de l'environnement et pour des raisons médicales, il est recommandé aux médecins d'inciter le plus possible leurs patients à utiliser des inhalateurs de poudre.

Seuls les groupes de patients suivants ne devraient pas utiliser d'inhalateur parce que la poudre ne parvient pas ou insuffisamment là où elle doit agir:

- patients dont le flux d'inspiration est faible

(<30 litres/min);

- nourrissons et enfants de moins de 4 ans;

- patients déments;

- patients traités en urgence ou intubés.

Pour ces patients, l'utilisation d'une solution inhalée ou d'un aérosol-doseur équipé d'une chambre reste recommandée.

Les principes actifs suivants sont autorisés en tant que préparation sans CFC par l’OICM (avril 2001):

\section{Tableau 1}

\begin{tabular}{lll}
\hline Classe de substance & Principe actif & $\begin{array}{l}\text { Préparations } \\
\text { disponibles sans CFC }\end{array}$ \\
\hline Stimulateurs $\beta-2$ & $\begin{array}{l}\text { Salbutamol } \\
\text { Fénotérol }\end{array}$ & $\begin{array}{l}\text { Airomir }{ }^{\circledR} \text {, Ventolin }{ }^{\circledR} \\
{ }^{\circledR}\end{array}$ \\
\hline Glucocotec ${ }^{\circledR}$
\end{tabular}

* La préparation ou sa version sans CFC n'a été autorisée que récemment et n'est pas encore commercialisée.

Informez vos patients sur le changement de gaz propulseur qui va se produire. Et n'hésitez pas à vous servir des feuilles d'information gratuites destinées aux patients, que vous pouvez vous procurer à l'adresse indiquée ci-dessous.

Expliquez bien que le changement de gaz propulseur ne pose généralement aucun problème. Le rapport utilité-risque des aérosols-doseurs ne contenant pas de CFC est identique à celui des aérosols en contenant, mais un changement de dose peut s'avérer nécessaire, selon la préparation et l'individu, et un suivi plus intensif s'impose donc pendant quelque temps (cf. texte informatif). De légers changements de goût sont également possibles.

\section{Remarques}

Pour plus d'informations concernant les aérosolsdoseurs sans CFC, consultez

- le site Internet de la Ligue pulmonaire suisse (www.lung.ch),

- le site Internet de l'OFEFP (www.environnementsuisse.ch).

Si vous souhaitez obtenir des feuilles d'information pour les patients (français, allemand, italien), commandez-les auprès de l'OCFIM: tél. 03132550 50, fax 03132550 58, e-mail: verkauf.zivil@bbl.admin.ch. 